\title{
Compostos bioativos e atividade antioxidante de variedades melhoradas de mamão
}

\author{
Bioactive compounds and antioxidant activity of improved varieties of papaya
}

\author{
Ronielli Cardoso Reis ${ }^{\mathrm{I}^{*}}$ Eliseth de Souza Viana ${ }^{\mathrm{I}}$ Jaciene Lopes de Jesus $^{\mathrm{I}}$ \\ Leonardo Franklin Lima ${ }^{I I}$ Tais Teixeira das Neves ${ }^{I I}$ Emerson Almeida da Conceição ${ }^{\text {III }}$
}

RESUMO

$O$ objetivo deste trabalho foi determinar a concentração de carotenoides totais, vitamina $C$, polifenóis totais e a atividade antioxidante de variedades melhoradas de mamão. Foram avaliadas quatro variedades melhoradas pertencentes ao grupo Solo (L60, L47-05, L47-08 e H54.78), duas pertencentes ao grupo Formosa (L33 e H36.45) e as variedades comerciais Tainung $n^{\circ} 1$ e Sunrise Solo. Dentre as variedades do grupo Solo, a linhagem L47-08 apresentou elevado teor de carotenoides totais e a variedade Sunrise Solo o maior conteúdo de vitamina C. Dentre as variedades do grupo Formosa, as variedades $L 33$ e H36.45 apresentaram elevada atividade antioxidante, e maiores teores, respectivamente, de polifenóis totais e vitamina $C$ do que a variedade comercial Tainung $n^{\circ} 1$. Os polifenóis totais e a vitamina $C$ apresentaram correlação significativa com a atividade antioxidante dos mamões avaliados.

Palavras-chave: Carica papaya, vitamina $C$, carotenoides $e$ polifenóis.

\section{ABSTRACT}

The objective of this research was to determine the concentration of carotenoids, vitamin $C$, polyphenols and antioxidant activity of improved varieties of papaya. Four improved varieties from Solo group (L60, L47-05, L47-08 and H54.78), two from Formosa group (L33 and H36.45) and commercial varieties Tainungn.1 and Sunrise Solo were evaluated. Among the Solo group varieties, L47-08 showed high levels of carotenoids and Sunrise Solo the highest content of vitamin C. Among the Formosa group varieties, H36.45 and L33 showed high levels of vitamin C and total polyphenols, as well as higher antioxidant activity. Total polyphenols and vitamin $C$ were significantly correlated with the antioxidant activity of papayas evaluated.

Key words: Carica papaya, vitamin C, carotenoids andpolyphenols.

\section{INTRODUÇÃO}

O consumo de frutas tropicais está aumentando no mercado nacional e internacional, devido ao reconhecimento das suas propriedades nutricionais e terapêuticas. O mamão destaca-se por apresentar elevado valor nutricional, sendo rico em açúcares, minerais e compostos bioativos como os carotenoides, vitamina $\mathrm{C}$ e polifenóis. $\mathrm{O}$ estudo de suas características é interessante por ser uma das frutas mais consumidas nas regiões tropicais e subtropicais do mundo, contribuindo expressivamente para o aporte de nutrientes e de compostos bioativos na dieta. Neste sentido, a Embrapa Mandioca e Fruticultura vem realizando pesquisas que visam à caracterização de variedades desenvolvidas pelo programa de melhoramento genético quanto ao teor de compostos bioativos, a fim de disponibilizar para a sociedade frutos com características iguais ou superiores às variedades comerciais.

Alguns compostos bioativos apresentam capacidade antioxidante e têm sido associados à proteção da saúde humana contra doenças degenerativas crônicas (LAKO et al., 2007). A capacidade antioxidante das frutas varia de acordo com os seus teores de vitamina $\mathrm{C}$, vitamina $\mathrm{E}$, carotenoides, flavonoides e outros compostos fenólicos (SAURA-CALIXTO \& GOÑI, 2006).

$A$ vitamina $C$ é um nutriente que previne doenças como escorbuto e desempenha um papel

\footnotetext{
'Laboratório de Ciência e Tecnologia de Alimentos, Embrapa Mandioca e Fruticultura, 44380-000, Cruz das Almas, BA, Brasil. E-mail: ronielli.reis@embrapa.br. *Autor para correspondência.

IIUniversidade Federal do Recôncavo da Bahia (UFRB), Cruz das Almas, BA, Brasil.

IIIFaculdade Maria Milza (FAMAN), Governador Mangabeira, BA, Brasil. 
importante como antioxidante biológico. O mamão apresenta concentrações que variam entre 42,5 e $142,6 \mathrm{mg} 100 \mathrm{~g}^{-1}$ de vitamina C (SANTANA et al., 2004; KUROZAWA, 2014). Os carotenoides são compostos que também apresentam propriedades antioxidantes e, dentre os carotenoides presentes no mamão, destacam-se o $\beta$-caroteno, licopeno e a $\beta$-criptoxantina (WALL, 2006). Os compostos fenólicos atuam como antioxidantes e, quando incorporados na alimentação, reduzem o risco de desenvolvimento de patologias, como arteriosclerose e câncer. Em mamão, constatou-se a presença dos compostos miricetina, fisetina, morina, quercetina, kaempferol e isorametina (LAKO et al., 2007).

Diferentes métodos têm sido utilizados para avaliar a capacidade antioxidante in vitro das frutas e os resultados obtidos variam de acordo com o método de análise empregado. Recomenda-se o uso de pelo menos dois métodos combinados para fornecer um resultado confiável da capacidade antioxidante total de um alimento (PÉREZ- JIMÉNEZ et al., 2008), sendo que os métodos FRAP, ABTS, DPPH e ORAC são os mais utilizados (CONTRERAS-CALDERÓN et al., 2011).

Diante do exposto, o objetivo deste trabalho foi caracterizar variedades melhoradas de mamão desenvolvidas pela Embrapa Mandioca e Fruticultura quanto ao teor de compostos bioativos e capacidade antioxidante.

\section{MATERIAL E MÉTODOS}

Delineamento experimental

As variedades de mamoeiro foram plantadas no delineamento em blocos casualizados, com quatro blocos. Para as avaliações, quatro frutos de cada bloco foram colhidos e as polpas homogeneizadas para compor a amostra, obtendo-se quatro amostras para cada variedade, caracterizando quatro repetições experimentais.

\section{Variedades avaliadas}

Foram avaliadas quatro variedades melhoradas pertencentes ao grupo Solo (L60, L4705, L47-08 e H54.78), duas pertencentes ao grupo Formosa (L33 e H36.45) e as variedades comerciais Tainung ${ }^{\circ} 1$ e Sunrise Solo. Os frutos foram colhidos no estádio 2 de maturação (frutos com $25 \%$ da superfície amarela) e analisados ao atingirem o estádio 5 (frutos com 76 a 100\% da superfície amarela).

Quantificação de polifenóis extraíveis totais e atividade antioxidante

Para a determinação do teor de polifenóis totais e da atividade antioxidante, extratos de mamão foram preparados, conforme descrito por LARRAURI et al. (1997), com modificações. A extração foi feita com metanol $50 \%$ por um período de 20 minutos em banho ultrassônico, seguida da centrifugação a $11.000 \mathrm{rpm}$ por 15 minutos. O resíduo foi submetido à segunda extração em acetona $70 \%$, seguindo o mesmo procedimento. Os extratos foram misturados e transferidos para o balão volumétrico de $50 \mathrm{~mL}$, completando-se o volume com água destilada.

Os polifenóis extraíveis totais foram determinados usando o reagente de Folin-Ciocalteau (1:3) e a curva padrão de ácido gálico, de acordo com o método descrito por SINGLETON et al. (1999) seguindo as modificações propostas por RUFINO et al. (2010).

A atividade antioxidante total foi determinada pela captura do radical $\mathrm{ABTS}^{+}$segundo metodologia proposta por RE et al. (1999). O decréscimo da absorbância foi medido após 6 minutos da reação e a leitura realizada a $734 \mathrm{~nm}$. Os resultados foram expressos como $\mu$ mol de Trolox $\mathrm{g}^{-1}$ de fruta, a partir da curva padrão de Trolox.

A determinação da atividade antioxidante foi também medida em função da atividade de sequestro do radical DPPH a partir do método descrito por BRAND-WILLIANS et al. (1995), com modificações propostas por RUFINO et al. (2010). $\mathrm{O}$ decréscimo da absorbância foi medido a $515 \mathrm{~nm}$ após 15 minutos de reação. A atividade antioxidante foi expressa em $\mathrm{CE}_{50}$ (quantidade de antioxidante necessária para reduzir em $50 \%$ a concentração inicial de DPPH) e em atividade de sequestro do radical livre DPPH (\% SRL), sendo calculado como:

$\% \mathrm{SRL}=[(\mathrm{C}-\mathrm{A}) / \mathrm{C}] \times 100$, em que: $\mathrm{C}$ é a absorbância do controle; A é a absorbância da amostra em solução.

Quantificação de carotenoides totais e vitamina C

Os teores de carotenoides totais foram determinados segundo RODRIGUEZ-AMAYA \& KIMURA (2004). O teor de vitamina C foi determinado por meio da reação do ácido ascórbico com o 2,6-diclorofenol indofenol (DCFI), conforme procedimento descrito por OLIVEIRA (2010). Todas as análises foram realizadas em triplicata.

Análise dos Resultados

Os dados foram submetidos à análise de variância (ANOVA) a 5\% de probabilidade, teste de Tukey a 5\% de significância e análise de correlação de Pearson com auxílio do programa estatístico SAS.

\section{RESULTADOS E DISCUSSÃO}

Dentro do grupo Solo, as variedades L4705, L47-08 e H54.78 não diferiram entre si $(\mathrm{P}>0,05)$ 
quanto ao teor de carotenoides totais (Tabela 1). A linhagem L47-08 apresentou teor de carotenoides totais superior $(28 \%)$ à variedade Sunrise Solo e a linhagem L60 apresentou o menor valor. Para o grupo Formosa, não houve diferença significativa $(\mathrm{P}>0,05)$ quanto aos teores de carotenoides totais e a média desse grupo foi semelhante à média do grupo Solo.

A variedade Sunrise Solo apresentou o maior conteúdo de vitamina $\mathrm{C}$ (Tabela 1), valor próximo ao obtido por LIM et al. (2007) para a mesma cultivar, que foi de $108 \mathrm{mg} 100 \mathrm{~g}^{-1}$.Já para o grupo Formosa, o híbrido H36.45 apresentou valor superior $(33,6 \%)$ ao Tainung $\mathrm{n}^{\circ} 1$. Entretanto, o teor de vitamina $C$ da variedade Tainung $\mathrm{n}^{\circ} 1$ foi inferior ao observado por OLIVEIRA et al. (2011), $80,2 \mathrm{mg} 100 \mathrm{~g}^{-1}$. Tais valores encontram-se dentro da faixa obtida por SANTANAet al (2004), que foi de 42,5 a 142,6mg 100g-1, em genótipos do Banco Ativo de Germoplasma da Embrapa Mandioca e Fruticultura.

Os valores de vitamina $\mathrm{C}$ dos mamões avaliados são semelhantes ao de outras frutas, como goiaba (85,9mg $100 \mathrm{~g}^{-1}$, OLIVEIRA et al., 2011) e açaí (84mg $100 \mathrm{~g}^{-1}$, RUFINO et al., 2010), e superior aos encontrados na laranja $\left(67 \mathrm{~g} 100 \mathrm{~g}^{-1}\right.$, LIM et al., 2007), fruta reconhecida como fonte de vitamina $C$, abacaxi (51mg $100 \mathrm{~g}^{-1}$, VIANA et al., 2013), manga $\left(17,5 \mathrm{mg} 100 \mathrm{~g}^{-1}\right.$, OLIVEIRA et al., 2011) e banana (4,9mg $100 \mathrm{~g}^{-1}$, LIM et al., 2007), que são frutas tão populares quanto o mamão e de alto consumo pela população. RAMFUL et al., (2011) classificaram as frutas em relação ao conteúdo de ácido ascórbico

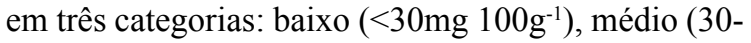

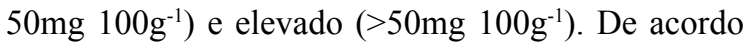
com essa classificação, todas asvariedades de mamão avaliadas podem ser classificadas como frutas com alto teor de ácido ascórbico.

Quanto ao teor de polifenóis totais (PET), dentro do grupo Solo, as variedades Sunrise Solo, L60, L47-05 e H54.78 não diferiram entre si em nível de $5 \%$ de significância (Tabela 1). Dentro do grupo Formosa, a linhagem L33 apresentou valor superior ao Tainung $\mathrm{n}^{\circ} 1$.Valores próximos foram constatados por ALMEIDA et al. (2011), 53,2mg EAG $100 \mathrm{~g}^{-1}$, para variedade do grupo Solo e por PATTHAMAKANOKPORN et al. (2008), 54mg EAG $100 \mathrm{~g}^{-1}$. Valores superiores foram relatados por OLIVEIRA et al. (2011) em mamão Formosa (88,1 mg EAG $\left.100 \mathrm{~g}^{-1}\right)$. Já LIM et al. (2007) e LAKO et al. (2007) encontraram valores inferiores, $28 \mathrm{mg}$ EAG $100 \mathrm{~g}^{-1}$ e $26 \mathrm{mg}$ EAG $100 \mathrm{~g}^{-1}$, respectivamente. $\mathrm{O}$ mamão apresentou maior conteúdo de PET do que a banana 'Nanica' e valores similares ao de outras frutas popularmente consumidas no Brasil como manga 'Tommy Atkins' e laranja.

Os valores da atividade antioxidante determinados pelos métodos DPPH e ABTS estão apresentados na figura 1. O híbrido H54.78 e a variedade comercial Sunrise Solo apresentaram os maiores percentuais de sequestro de radical livre (\% SRL), 25,59 e 23,77\%, respectivamente (Figura 1A), e baixos valores de $\mathrm{CE}_{50}$. Para os genótipos do grupo Formosa (Figura 1B), a linhagem L33 e o híbrido

Tabela 1- Carotenoides totais, vitamina C e polifenóis extraíveis totais (PET) em variedades melhoradas de mamão.

\begin{tabular}{|c|c|c|c|c|}
\hline & Variedades & Carotenoides totais $\left(\mu \mathrm{g} \mathrm{g}^{-1}\right)$ & Vitamina C (mg $\left.100 \mathrm{~g}^{-1}\right)$ & PET (mg EAG $100 \mathrm{~g}^{-1}$ ) \\
\hline \multirow{5}{*}{ Grupo Solo } & Sunrise Solo & $41,30^{\mathrm{b}}$ & $114,52^{\mathrm{a}}$ & $78,41^{\mathrm{a}}$ \\
\hline & L60 & $29,00^{\mathrm{c}}$ & $75,16^{\mathrm{bc}}$ & $61,86^{\mathrm{ab}}$ \\
\hline & L47-05 & $44,62^{\mathrm{ab}}$ & $60,38^{\mathrm{c}}$ & $56,94^{\mathrm{ab}}$ \\
\hline & L47-08 & $52,88^{\mathrm{a}}$ & $66,79^{\mathrm{c}}$ & $46,46^{\mathrm{b}}$ \\
\hline & H54.78 & $46,41^{\mathrm{ab}}$ & $89,43^{b}$ & $75,70^{\mathrm{a}}$ \\
\hline \multirow{7}{*}{ Grupo Formosa } & Média & $42,84 \pm 8,81$ & $81,25 \pm 21,54$ & $63,87 \pm 13,29$ \\
\hline & $\mathrm{CV}(\%)$ & 20,57 & 26,50 & 20,81 \\
\hline & Tainung $\mathrm{n}^{\mathrm{o}} \mathbf{1}$ & $42,15^{\mathrm{ns}}$ & $70,09^{b}$ & $63,00^{\mathrm{b}}$ \\
\hline & L33 & $44,13^{\mathrm{ns}}$ & $85,45^{\mathrm{ab}}$ & $76,27^{\mathrm{a}}$ \\
\hline & H36.45 & $37,05^{\mathrm{ns}}$ & $93,64^{\mathrm{a}}$ & $68,08^{a b}$ \\
\hline & Média & $41,11 \pm 3,65$ & $83,19 \pm 11,96$ & $69,12 \pm 6,70$ \\
\hline & CV (\%) & 8,89 & 14,39 & 9,69 \\
\hline
\end{tabular}

${ }^{\mathrm{ns}}$ não significativo $(\mathrm{P}>0,05) ; \mathrm{PET}=$ polifenóis extraíveis totais; EAG: equivalente em ácido gálico. 


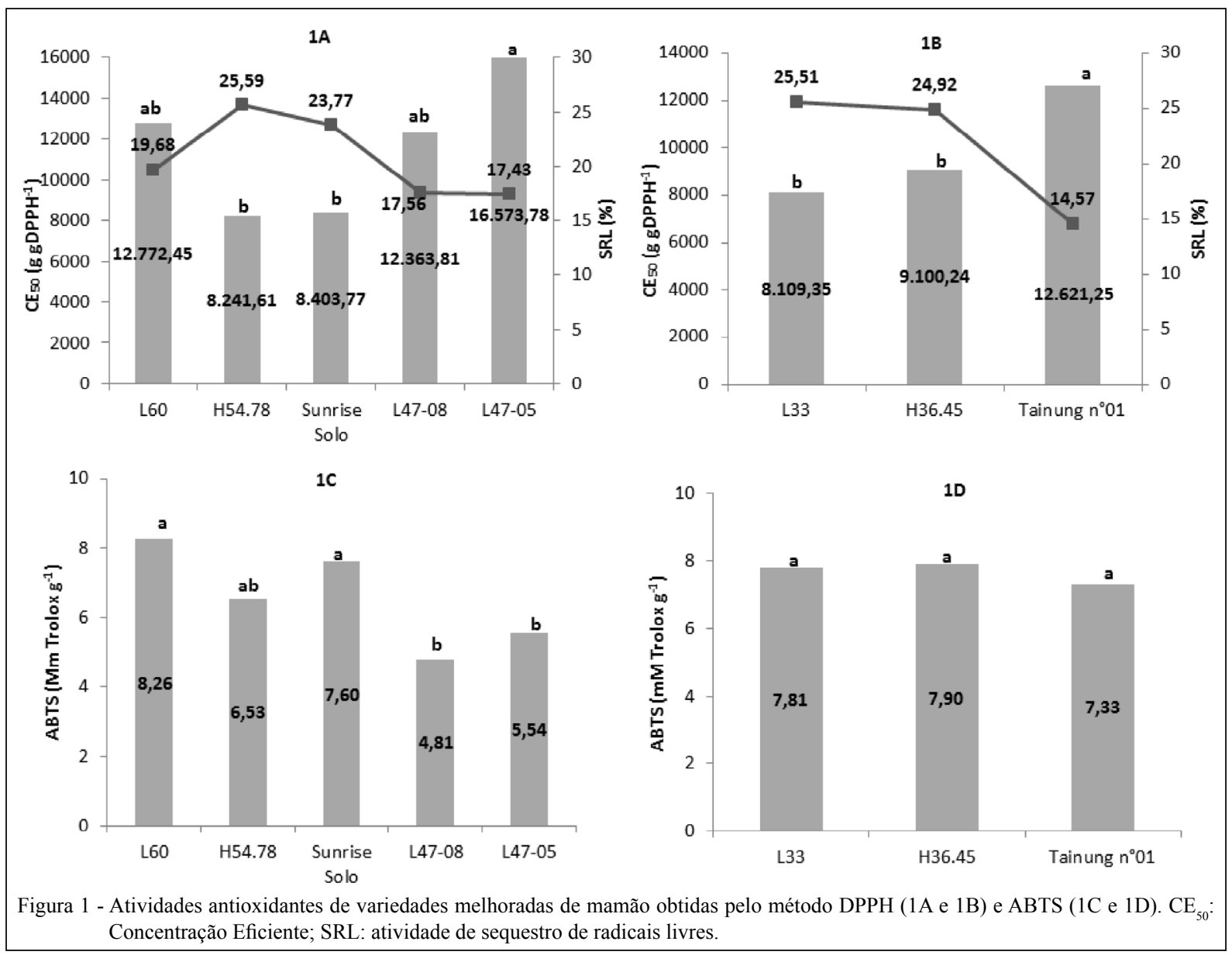

H36.45 apresentaram os menores valores de $\mathrm{CE}_{50}$ e maior potencial antioxidante, 25,51 e $24,92 \%$ de SRL respectivamente, sendo superiores à variedade comercial Tainung $\mathrm{n}^{\circ} 1$.

Pelo método ABTS, para os genótipos do grupo Solo (Figura 1C), os maiores valores de atividade antioxidante foram verificados para as variedades Sunrise Solo, L60 e H54.78. Para o grupo Formosa (Figura 1D), não se constatou diferença significativa $(\mathrm{P}>0,05)$ entre os três genótipos avaliados, e a atividade antioxidante variou de 7,33 a $7,90 \mu \mathrm{M}$ Trolox $\mathrm{g}^{-1}$. Resultado semelhante foi obtido por ALMEIDAet al. (2011), 7,60 $\mu \mathrm{M}$ Trolox g-1 em mamão. Os oito genótipos de mamão avaliados apresentaram maiores atividades antioxidantes, quando comparados com outras frutas, como jaca, abacaxi, sapoti e umbu (ALMEIDA et al., 2011).

A atividade antioxidante determinada pelo método ABTS não apresentou correlação positiva significativa com nenhum composto. Correlação negativa significativa $(\mathrm{R}=-0,6835, \quad \mathrm{P}<0,01) \quad$ foi encontrada entre o teor de PET e o $\mathrm{CE}_{50}$, indicando que frutos com maiores teores de PET apresentaram maiores atividades antioxidantes, uma vez que, para o $\mathrm{CE}_{50}$, essa relação é inversa. RUFINO et al. (2010) também verificaram correlações negativas entre o teor de PET e o $\mathrm{CE}_{50}(\mathrm{R}=-0,72)$ ao avaliar 18 frutas tropicais. Várias pesquisas têm correlacionado o conteúdo de polifenóis presentes nas frutas e a atividade antioxidante, usando diferentes métodos de análise. ALMEIDA et al. (2011) verificaram correlações positivas entre o teor de PET e a atividade antioxidante em frutas exóticas do nordeste do Brasil, utilizando os métodos ABTS e DPPH. RODRIGUES et al. (2011) também verificaram correlações positivas em cultivares de mirtilo produzidas no Brasil, utilizando os dois métodos. Entretanto, no presente estudo, foi verificada apenas correlação significativa entre o teor de PET e a atividade antioxidante utilizando o método DPPH.

Correlação positiva significativa foi encontrada entre o teor de vitamina $\mathrm{C}$ e PET $(\mathrm{R}=0,7249, \mathrm{P}<0,01)$ e, portanto, frutos com maiores teores de vitamina $\mathrm{C}$ apresentaram maiores teores de PET. RUFINO et al. (2010) também verificaram 
correlações positivas entre PET e vitamina C $(\mathrm{R}=0,70)$, ao avaliar 18 frutas tropicais. Assim como o PET, o teor de vitamina $\mathrm{C}$ correlacionouse negativamente com o $\mathrm{CE}_{50}(-0,7045, \mathrm{P}<0,01)$, indicando que frutos com maiores teores de vitamina $\mathrm{C}$ apresentaram maiores atividades antioxidantes. Elevadas correlações entre a atividade antioxidante e o teor de vitamina $\mathrm{C}$ foram encontradas para frutas cítricas (ZULUETA et al., 2007) e goiabas (THAIPONG et al., 2006). Entretanto, alguns trabalhos relatam correlação negativa entre o teor de vitamina $\mathrm{C}$ e a atividade antioxidante (PANTELIDIS et al., 2007), ou não observam correlação significativa (GIL et al., 2002; ALMEIDA et al., 2011). Vale ressaltar que esses resultados são dependentes dos métodos de análise utilizados.

Os resultados do presente estudo indicam que os compostos fenólicos e a vitamina $\mathrm{C}$ contribuem significativamente para a atividade antioxidante dos mamões avaliados. Entretanto, a atividade antioxidante não pode ser atribuída somente aos conteúdos de vitamina $\mathrm{C}$ e polifenóis presentes no mamão, mas também a outros compostos antioxidantes. $\mathrm{O}$ fato de não terem sido verificadas correlações positivas significativas entre a atividade antioxidante e os teores de carotenoides não significa que tais compostos não contribuem para essa atividade. Esse resultado pode ser devido aos métodos de análise utilizados ou ser decorrente de mecanismos de sinergismo e antagonismo entre tais compostos ainda desconhecidos.

\section{CONCLUSÃO}

As variedades L33 e H36.45, do grupo Formosa, apresentaram elevada atividade antioxidante, e maiores teores, respectivamente, de polifenóis totais e vitamina $\mathrm{C}$ do que a variedade comercial Tainung $\mathrm{n}^{\circ} 1$. Os compostos fenólicos e a vitamina $\mathrm{C}$ contribuíram significativamente para a atividade antioxidante dos mamões avaliados.

\section{AGRADECIMENTOS}

À Fundação de Amparo à Pesquisa do Estado da Bahia (FAPESB), ao Conselho Nacional de Desenvolvimento Científico e Tecnológico $(\mathrm{CNPq})$ e à Embrapa, pelo suporte financeiro para a realização da pesquisa.

\section{REFERÊNCIAS}

ALMEIDA, M.M.B. et al. Bioactive compounds and antioxidant activity of fresh exotic fruits from northeastern Brazil. Food Research International, v.44, p.2155-2159, 2011. Disponível em: <http://
www.sciencedirect.com/science/article/pii/S0963996911002109>. Acesso em: 20 jan. 2013. doi: 10.1016/j.foodres.2011.03.051.

AMORIM, E.P. et al. Caracterização de acessos de bananeira com base na concentração de compostos funcionais. Ciência Rural, v.41, n.4, p.592-598, 2011. Disponível em: <http://www.scielo. br/pdf/cr/v41n4/a939cr2352.pdf>. Acesso em: 30 jan. 2013. doi: 10.1590/S0103-84782011005000042.

BRAND-WILLIAMS W. et al. Use of a free radical method to evaluate antioxidant activity. Food Science and Technology, v.28, n.1, p.25-30, 1995. Disponível em: <http://www.sciencedirect. com/science/article/pii/S0023643895800085>. Acesso em: 27 jul. 2013. doi: 10.1016/S0023-6438(95)80008-5.

CONTRERAS-CALDERÓN, J. et al. Antioxidant capacity, phenolic content and vitamin $\mathrm{C}$ in pulp, peel and seed from 24 exotic fruits from Colombia. Food Research International, v.44, p.2047-2053, 2011. Disponível em: <http://www.sciencedirect. com/science/article/pii/S0963996910004357>. Acesso em: 02 mar. 2013. doi: 10.1016/j.foodres.2010.11.003.

GIL, M.I. et al. Antioxidant capacities, phenolic compounds, carotenoids, and vitamin $\mathrm{C}$ contents of nectarine, peach, and plum cultivars from California. Journal of the Science of Food and Agriculture, v.50, p.4976-4982, 2002. Disponível em: <http:// ucce.ucdavis.edu/files/datastore/234-25.pdf>. Acesso em: $30 \mathrm{abr}$. 2013. doi: 10.1021/jf020136b.

KUROZAWA, L. E. et al. Ascorbic acid degradation of papaya during drying: effect of process conditions and glass transition phenomenon. Journal of Food Engineering, v.123, p.157-164, 2014. Disponível em: <http://www.sciencedirect.com/science/ article/pii/S0260877413004524>. Acesso em: 25 mar. 2014. doi: 10.1016/j.jfoodeng.2013.08.039.

LAKO, J. et al. Phytochemical flavonols, carotenoids and the antioxidant properties of a wide selection of Fijian fruit, vegetables and other readily available foods. Food Chemistry, v.101, p.17271741, 2007. Disponível em: <http://www.sciencedirect.com/ science/article/pii/S0308814606000872>. Acesso em: 05 ago. 2013. doi: 10.1016/j.foodchem.2006.01.031.

LARRAURI, J.A et al. Effect of drying temperature on the stabilitity of polyphenols and antioxidant activity of red grape pomace peels. Journal Agriculture and Food Chemistry, v.45, p.13901393, 1997. Disponível em: <http://pubs.acs.org/doi/abs/10.1021/ jf960282f>. Acesso em: 10 abr. 2013. doi: 10.1021/jf960282f.

LIM, Y.Y. et al. Antioxidant properties of several tropical fruits: a comparative study. Food Chemistry, v.103, n.3, p.1003-1008, 2007. Disponível em: <http://www.sciencedirect.com/science/ article/pii/S0308814606007825>. Acesso em: 03 fev. 2014. doi: 10.1016/j.foodchem.2006.08.038.

OLIVEIRA, D.S. et al. Vitamina C, carotenoides, fenólicos totais e atividade antioxidante de goiaba, manga e mamão procedentes da Ceasa do Estado de Minas Gerais. Acta Scientiarum Health Sciences, v.33, n.1, p.89-98, 2011. Disponível em: $<$ http://periodicos.uem.br/ojs/index.php/ActaSciHealthSci/ article/view/8052>. Acesso em: 26 abr. 2012. doi: 10.4025/ actascihealthsci.v33i1.8052.

OLIVEIRA, L.A. Manual de laboratório: análises físico-químicas de frutas e mandioca. Cruz das Almas: Embrapa Mandioca e Fruticultura, 2010. 248p. 
PANTELIDIS, G.E. et al. Antioxidant capacity, phenol, anthocyanin and ascorbic acid contents in raspberries, blackberries, red currants, gooseberries and Cornelian cherries. Food Chemistry, v.102, p.777-783, 2007. Disponível em: <http://www.sciencedirect.com/ science/article/pii/S0308814606004833>. Acesso em: 25 abr. 2014. doi: 10.1016/j.foodchem.2006.06.021.

PATTHAMAKANOKPORN, O. et al. Changes of antioxidant activity and total phenolic compounds during storage of selected fruits. Journal of Food Composition and Analysis, v.21, p.241248, 2008. Disponível em: <http://www.sciencedirect.com/ science/article/pii/S088915750700172X>. Acesso em: 06 abr. 2014. doi: $10.1016 /$ j.jfca.2007.10.002.

PÉREZ-JIMÉNEZ, J. et al.Updated methodology to determine antioxidant capacity in plant foods, oils and beverages: extraction, measurement and expression of results. Food Research International, v.41, p.272-285, 2008. Disponível em: $<$ http://www. sciencedirect.com/science/article/pii/S088915750700172X>. Acesso em: mar. 2014. doi: 10.1016/j.jfca.2007.10.002.

RAMFUL, D. et al. Polyphenol composition, vitamina $\mathrm{C}$ content and antioxidant capacity of Mauritian citrus fruit pulps. Food Research International, v.44, p.2088-2099, 2011. Disponível em: <http:// www.sciencedirect.com/science/article/pii/S0963996911002158>. Acesso em: 30 jun. 2013. doi: 10.1016/j.foodres.2011.03.056.

RE, et al. Antioxidant activity applying improved ABTS radical cation decolorization assay. Free Radical Biology and Medicine, v.26, p.1231-1237, 1999. Disponível em: <http://www.ncbi.nlm. nih.gov/pubmed/10381194>. Acesso em: 02 jan. 2011.

RODRIGUES, E. et al. Phenolic compounds and antioxidant activity of blueberry cultivars grown in Brazil. Ciência e Tecnologia de Alimentos, v.31, n.4, p.911-917, 2011 Disponível em: $<$ http://www.scielo.br/scielo.php?pid=S0101$20612011000400013 \&$ script $=$ sci arttext $>$. Acesso em: 25 mar. 2013. doi: $10.1590 / \mathrm{S} 0101-20612011000400013$.

RODRIGUEZ-AMAYA, D.; KIMURA, M. Harvest plus handbook for carotenoid analysis. Washington, DC and Cali: IFPRI and CIAT, 2004. 58p.

RUFINO, M.S.M. et al. Bioactive compounds and antioxidant capacities of 18 non-traditional tropical fruits from Brazil. Food Chemistry, v.121, p.996-1002, 2010. Disponível em: <http:// www.sciencedirect.com/science/article/pii/S0308814610001172>. Acesso em: abr. 2011. doi:10.1016/j.foodchem.2010.01.037.
SANTANA, R.R. et al. Genótipos melhorados de mamão (Carica papaya L.,): avaliação sensorial e físico-química dos frutos. Ciência e Tecnologia de Alimentos, v.24, n.2, p.217-222, 2004. Disponível em: $<\mathrm{http} / /$ www.scielo.br/scielo.php?pid=S010120612004000200010\&script=sci_arttext $>$. Acesso em: abr. 2011. doi: 10.1590/S0101-20612004000200010.

SAURA-CALIXTO, F.; GOÑI, I. Antioxidant capacity of the Spanish Mediterranean diet. Food Chemistry, v.94, p.442-447, 2006. Disponível em: <http://www.sciencedirect.com/science/ article/pii/S0308814604008829>. Acesso em: mar. 2014. doi: 10.1016/j.foodchem.2004.11.033.

SINGLETON, V.L. et al. Analysis of total phenols and other oxidation substrates and antioxidants by means of Folin-Ciocalteu reagent. Methods in Enzymology, v.299, p.152-178, 1999. Disponível em: <http://www.sciencedirect.com/science/article/pii/ S0076687999990171>. Acesso em: 20 ago. 2013. doi: 10.1016/ S0076-6879(99)99017-1.

THAIPONG, K. et al. Comparison of ABTS, DPPH, FRAP, and ORAC assays for estimating antioxidant activity from guava fruit extracts. Journal of Food Composition and Analysis, v.19, p.669-675, 2006. Disponível em: <http://www.sciencedirect.com/ science/article/pii/S08891575060000>. Acesso em: 30 jun. 2014. doi: 10.1016/j.jfca.2006.01.003

VIANA, E.S. et al. Caracterização físico-química de novos híbridos de abacaxi resistentes à fusariose. Ciência Rural, v.43, n.7, p.1155-1161, 2013. Disponível em: <http://www.scielo. br/scielo.php?pid=S0103-84782013000700003\&script $=$ sci arttext>. Acesso em: 23 ago. 2013. doi: 10.1590/S010384782013005000075 .

WALL, M.M. Ascorbic acid, vitamin A, and mineral composition of banana (Musa sp.) and papaya (Carica papaya) cultivars grown in Hawaii. Journal of Food Composition and Analysis, v.19, p.434-445, 2006. Disponível em: <http://www.sciencedirect.com/ science/article/pii/S088915750600007X $>$. Acesso em: 15 abr. 2013. doi: 10.1016/j.jfca.2006.01.002.

ZULUETA, A. et al. Vitamin C, vitamin A, phenolic compounds and total antioxidant capacity of new fruit juice and skim milk mixture beverages marketed in Spain. Food Chemistry, v.103, p.1365-1374, 2007. Disponível em: <http://www.sciencedirect. com/science/article/pii/S0308814606008363>. Acesso em: 12 jun. 2013. doi: $10.1016 /$ j.foodchem.2006.10.052. 